\title{
Atmospheric image blur with finite outer scale or partial adaptive correction
}

\author{
P. Martinez ${ }^{1}$, J. Kolb ${ }^{1}$, A. Tokovinin ${ }^{2}$, and M. Sarazin ${ }^{1}$ \\ ${ }^{1}$ European Southern Observatory, Karl-Schwarzschild-Strasse 2, 85748 Garching, Germany \\ e-mail: patrice.martinez@obspm.fr \\ 2 Cerro-Tololo Inter American Observatory, Casilla 603, La Serena, Chile
}

Received 12 March 2010 / Accepted 22 March 2010

ABSTRACT

\begin{abstract}
Context. Seeing-limited resolution in large telescopes operating over wide wavelength ranges depends substantially on the turbulence outer scale and cannot be adequately described by one seeing value.

Aims. This study attempts to clarify frequent confusions between seeing and the full-width at half-maximum of long-exposure images in large telescopes, also often called delivered image quality.

Methods. We study the effects at the focus of a telescope of finite turbulence outer scale and partial adaptive corrections, both corresponding to a reduction in the low-frequency content of the phase perturbation spectrum, by means of analytical calculations and numerical simulations.

Results. If a von Kàrmàn turbulence model is adopted, a simple approximate formula predicts the dependence of atmospheric longexposure resolution on the outer scale over the entire practically interesting range of telescope diameters and wavelengths. In the infrared (IR), the difference from the standard Kolmogorov seeing formula can exceed a factor of two. We find that a low-order adaptive turbulence correction produces residual wavefronts effectively of small outer scale, so even a very low compensation order leads to a substantial improvement in resolution over seeing, compared to the standard theory.

Conclusions. Seeing-limited resolution of large telescopes, especially in the IR, is currently under estimated by not accounting for the outer scale. On the other hand, adaptive-optics systems optimized for diffraction-limited imaging in the IR can improve the resolution in the visible by as much as a factor of two.
\end{abstract}

Key words. techniques: high angular resolution - instrumentation: high angular resolution - telescopes

\section{Introduction}

Image blur of astronomical objects caused by terrestrial atmosphere is traditionally called "seeing". In the 2nd half of the 20th century this phenomenon was understood and quantified (Young 1974). This understanding was based on considering the distorted wavefronts as a random stationary process with a power-law spectrum - the Kolmogorov-Obukhov model (Tatarskii 1961; Roddier 1981). This theory describes the shape of the atmospheric long-exposure point spread function (PSF) and many other phenomena by a single parameter, e.g; the Fried's coherence radius $r_{0}$ (Fried 1966). The theory predicts the dependence of the PSF full-width at half maximum $(F W H M) \varepsilon_{0}$ on wavelength $\lambda$ and $r_{0}$ to be

$\varepsilon_{0}=0.976 \lambda / r_{0}$.

In this paper, we assume that $r_{0}$ and $\varepsilon_{0}$ refer to observations at zenith. By adopting a standard wavelength $\lambda=500 \mathrm{~nm}$, we can replace $r_{0}$ with $\varepsilon_{0}$ and this single parameter is nowadays usually called "seeing". Here we use the term seeing in this precise sense, meaning $\varepsilon_{0}$ at $500 \mathrm{~nm}$ at zenith

The success of this theory led most people to believe that the atmospheric parameters $r_{0}$ or $\varepsilon_{0}$ actually exist and can be measured to high accuracy, given adequate means. The match between real physical quantities such as PSF or various statistical estimates of distorted wavefronts to the Kolmogorov-Obukhov theory varies from very good to poor, but it is never perfect. The concept of seeing becomes questionable if we push it too far.

The physics of turbulence implies that the spatial power spectral density (PSD) of phase distortions $W_{\phi}(f)(f$ is the spatial frequency in $\mathrm{m}^{-1}$ ) deviates from the pure power law at low frequencies. A popular von Kàrmàn $(\mathrm{vK})$ turbulence model (Tatarskii 1961; Ziad et al. 2000; Conan 2000) introduces an additional parameter, the outer scale $L_{0}$

$W_{\phi}(\boldsymbol{f})=0.0229 r_{0}^{-5 / 3}\left(|\boldsymbol{f}|^{2}+L_{0}^{-2}\right)^{-11 / 6}$.

Equation (2) is the definition of $L_{0}$. The Kolmogorov model corresponds to $L_{0}=\infty$. In the vK model, $r_{0}$ describes the highfrequency asymptotic behavior of the spectrum, and thus loses its sense of an equivalent wavefront coherence diameter as defined originally by Fried (1966). Obviously, Eq. (1) is no longer valid as well.

It remains an open question whether wavefront statistics actually correspond to Eq. (2). Proving the vK model experimentally would be a difficult and eventually futile goal because large-scale wavefront perturbations are anything but stationary. However, it is firmly established that the phase spectrum does deviate from a power law (Ziad et al. 2000; Tokovinin et al. 2007). Equation (2) with an additional parameter $L_{0}$ provides a useful first-order description of this behavior. Existing experimental data on $L_{0}$ are interpreted here in this sense. 
In this paper, we study the modifications of Eq. (1) implied by the finite outer scale. Our analytical calculations are confirmed by extensive numerical simulations. We show that for finite $L_{0}$ the atmospheric $F W H M$ becomes smaller than predicted by Eq. (1), and that this difference can be substantial. The practical consequences for operation of large telescopes are discussed. The lack of low-frequency power is typical not only for the vK turbulence, but also for partially corrected wave-fronts resulting, e.g., from tip-tilt compensation (fast guiding) or low-order adaptive-optics (AO) correction. This correction leads to a small effective $L_{0}$. We apply the same analytical treatment to this case and study the shrinking of the PSF halo under partial AO compensation.

\section{Analytical treatment}

The calculation of the long-exposure PSF is performed by multiplying the telescope optical transfer function (OTF) by an additional term, the atmospheric OTF

$T_{a}(\boldsymbol{u})=\exp \left[-0.5 D_{\phi}(\lambda \boldsymbol{u})\right]$,

where $\boldsymbol{u}$ is the angular spatial frequency (in inverse radians), $\lambda$ is the imaging wavelength, and $D_{\phi}(\boldsymbol{r})$ is the phase structure function (SF) (Goodman 1985; Roddier 1981). This expression is in general, applicable to any turbulence spectrum and any telescope diameter. In the case of a large ideal telescope with diameter $D \gg r_{0}$, the diffraction can be neglected and the long-exposure OTF and PSF are accurately described by Eq. (3).

The analytic expression for the phase structure function in the von Kàrmàn model can be found in Conan (2000), Consortini et al. (1972), and Tokovinin (2002). For infinite $L_{0}$, it transforms into $D_{\phi}(r)=6.88\left(r / r_{0}\right)^{5 / 3}$.

Figure 1 (top) plots the SFs for Kolmogorov and vK models with the same $r_{0}$. In the latter case, the SF saturates at $r>L_{0}$, reaching asymptotically the level $0.17\left(L_{0} / r_{0}\right)^{5 / 3}$. It reaches halfsaturation at $r=0.17 L_{0}$. The Kolmogorov SF with the same $r_{0}$ crosses the vK saturation level at $r=0.109 L_{0}$. This tells us that the effect of a finite outer scale is strong at distances much shorter than $L_{0}$, and that it would be misleading to compare $L_{0}$ directly with the telescope diameter.

Putting the vK SF into Eq. (3), we find that for finite $L_{0}$, $T_{a}(\boldsymbol{u})$ does not go to zero at large arguments, therefore its inverse Fourier transform (the PSF) formally does not exist. However, when $r_{0} \ll L_{0}$ this level is small and can be neglected. In Fig. 1 (bottom), we compare the PSF profiles for different values of $L_{0} / r_{0}$, including $L_{0}=\infty$.

A first-order approximation of the $F W H M$ of atmospheric PSFs $\left(\varepsilon_{\mathrm{vK}}\right)$ under vK turbulence was proposed by Tokovinin (2002) to be

$\varepsilon_{\mathrm{vK}} \approx \varepsilon_{0} \sqrt{1-2.183\left(r_{0} / L_{0}\right)^{0.356}}$.

This formula is valid for $L_{0} / r_{0}>20$ to an accuracy of $\pm 1 \%$. We recall the reader that while $r_{0}$ depends on the wavelength, $L_{0}$ does not. At smaller $L_{0} / r_{0}$ values, the atmospheric PSF develops a strong core-halo structure, and its $F W H M$ becomes less and less meaningful. The actual PSF in a telescope is a convolution of the atmospheric blur with diffraction, aberrations, guiding errors, etc. As neither of these factors is described by a Gaussian, calculation of the combined FWHM as a quadratic sum of individual contributions is not accurate.

A formula for the $F W H E$, full-width at half-energy $\left(\beta_{v k}\right)$, can be derived with the same accuracy (Tokovinin 2002)

$\beta_{\mathrm{vK}} \approx \beta_{0} \sqrt{1-1.534\left(r_{0} / L_{0}\right)^{0.347}}$,
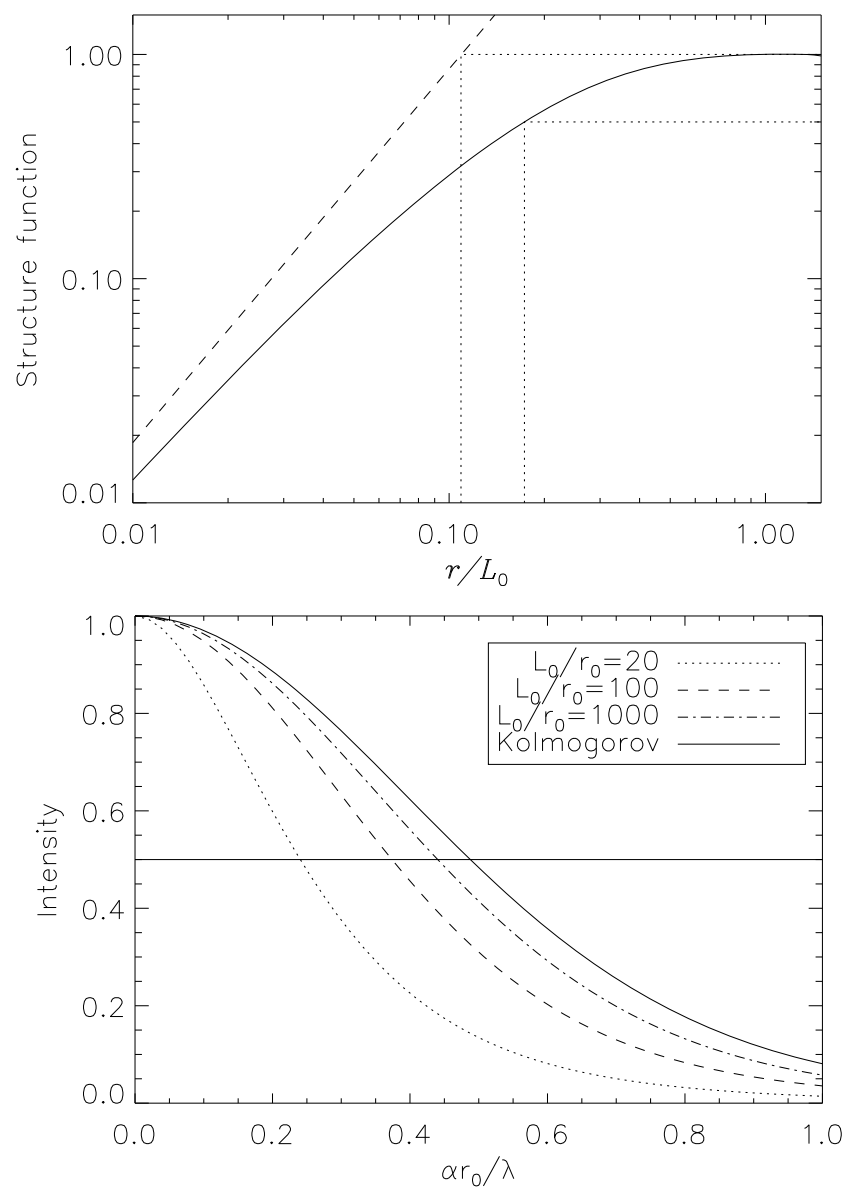

Fig. 1. Top panel: comparison of the von Kàrmàn (full line) and Kolmogorov (dashed line) phase structure functions with the same $r_{0}$. Bottom panel: normalized atmospheric PSFs for different $L_{0} / r_{0}$ ratios.

where $\beta_{0}$ is the diameter of the circle that contains one-half of the total PSF energy in the Kolmogorov theory $\left(\beta_{0}=1.15 \lambda / r_{0}\right)$.

The following section gathers results obtained with extensive numerical simulations to confirm the reliability and validity domain of Eq. (4), and thereby Eq. (5).

\section{Numerical simulations}

\subsection{Random wavefronts}

The atmospheric turbulence was generated with 1000 uncorrelated phase screens on a $8192 \times 8192$ array equivalent to a $100 \mathrm{~m}$ width physical size (pixel size $12.2 \mathrm{~mm}$ ). The principle of the generation of a phase screen is based on the Fourier approach (McGlamery 1976): randomized white noise maps are colored in the Fourier space by the turbulence PSD (Eq. (2)), and the inverse Fourier transform of an outcome corresponds to a phase screen realization. The large size of the simulated phase screens is mandatory for correctly sampling the $L_{0}$ and computing PSF for large telescopes. The simulations considered several $L_{0}$ cases $(10,22,32.5,50,65 \mathrm{~m}$, and $\infty)$.

Several investigations were carried out on the phase screens to ascertain that their statistics indeed correspond to the input parameters $r_{0}$ and $L_{0}$. For example, we compared the phase variance, and the variances in the first 100 Zernike coefficients for $D=42 \mathrm{~m}\left(r_{0}=12.12 \mathrm{~cm}\right.$ and $\left.L_{0}=65 \mathrm{~m}\right)$ with their expected values given by Conan (2000) and found good agreement. The 
P. Martinez et al.: Atmospheric image blur with finite outer scale or partial adaptive correction
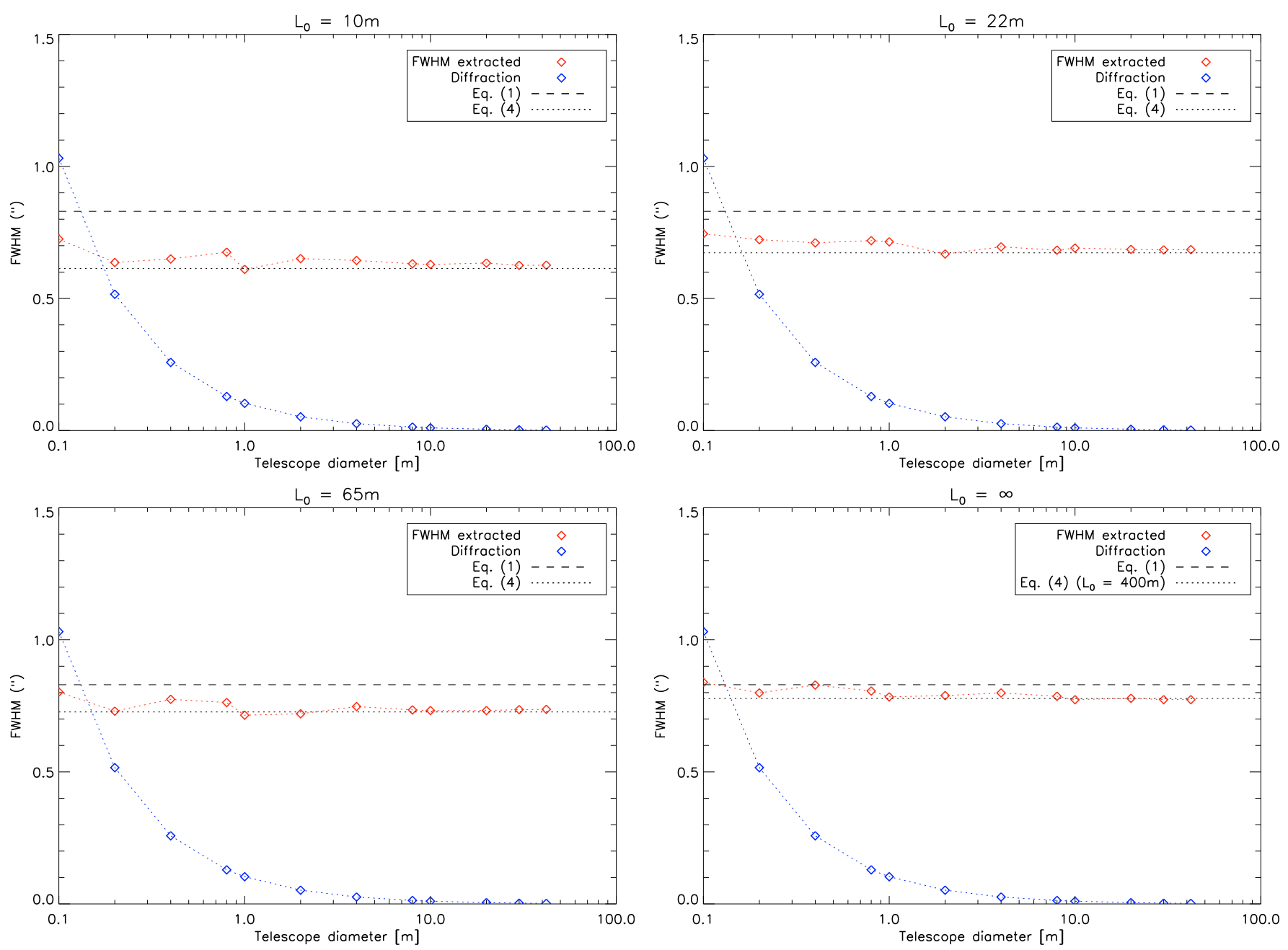

Fig. 2. The atmospheric $F W H M$ of simulated long-exposure PSFs $\theta_{\mathrm{a}}$ versus telescope diameter for several $L_{0}$ values $\left(\lambda=0.5 \mu \mathrm{m}, \varepsilon_{0}=0.83^{\prime \prime}\right)$. The blue curves trace the diffraction $F W H M \theta_{\text {dif }}=\lambda / D$.

phase variance matches expectations to within $1.7 \%$, while the variance in the tip and tilt components agrees with their theoretical values to within 1.3 and $0.7 \%$, respectively. The case of $L_{0}=\infty$ is particular: the variance in the tip and tilt coefficients does not fit their theoretical values, corresponding instead to a finite outer scale in the range of $200 \mathrm{~m}$ to $500 \mathrm{~m}$. This is a consequence of the finite size of the simulated phase screens.

Several telescope diameters were considered ranging from $10 \mathrm{~cm}$ to $42 \mathrm{~m}$. The wavelength domain ranges from the $U$-band to $M$-band, while the seeing ranges from $0.1^{\prime \prime}$ to $1.8^{\prime \prime}$.

All simulations adopt fast Fourier transforms (FFT) of $8192 \times 8192$ arrays to generate the long-exposure PSFs (over 1000 realizations). The same set of phase screens is used for all telescope diameters. As a result of the very large arrays involved in handling both phase screen statistics and aliasing effect (e.g., large-aperture cases), small telescope diameters $(<1 \mathrm{~m})$ may be affected by coarse pupil sampling. The effect of speckle structure is also stronger for small $D$, causing a larger random scatter in the results.

\subsection{Measurement of the FWHM}

We determined the $F W H M$ of the simulated long-exposure PSFs $\theta_{\mathrm{PSF}}$ in the following way. The PSFs were first azimuthally averaged. The 10th order polynomial was then fitted to this curve, and the radius where it crosses the $1 / 2$ of the maximum intensity was determined. The results of this routine were then compared with those of another algorithm (Kolb 2005) applied to the same set of PSFs, and both gave similar values (e.g., \pm 1 pixel for $D=$ $8 \mathrm{~m}$ and $L_{0}=22 \mathrm{~m}$; i.e., $\left.0.006^{\prime \prime}\right)$.

The simulated PSFs are broadened by diffraction and are thus not directly comparable to Eq. (4). We approximately account for this by subtracting quadratically the diffraction $F W H M$ $\theta_{\text {dif }}=\lambda / D$ evaluating the true width to be

$\theta_{\mathrm{a}} \approx \sqrt{\theta_{\mathrm{PSF}}^{2}-\theta_{\mathrm{dif}^{2}}}$

This provides a good approximation of $\varepsilon_{\mathrm{VK}}$ as long as the diffraction blur is small, $D \gg r_{0}$, but cannot be applied to small diameters, as mentioned above, because the individual broadening factors are not Gaussian. This explains why our results for small $D$ are inaccurate.

\subsection{Outer scale and telescope diameter}

The first series of simulations aims at defining the general trend of atmospheric $F W H M \theta_{\mathrm{a}}$ in large telescopes in the presence of finite outer scale. We compare $\theta_{\mathrm{a}}$ to Eq. (4) and to the seeing $\varepsilon_{0}$, fixed to $0.83^{\prime \prime}$ in this case. Some results are presented in Fig. 2.

From Fig. 2, it is straightforward to see that $\theta_{\mathrm{a}}<\varepsilon_{0}$ in all cases, even for $L_{0}=\infty$ because all simulated wave-fronts have 

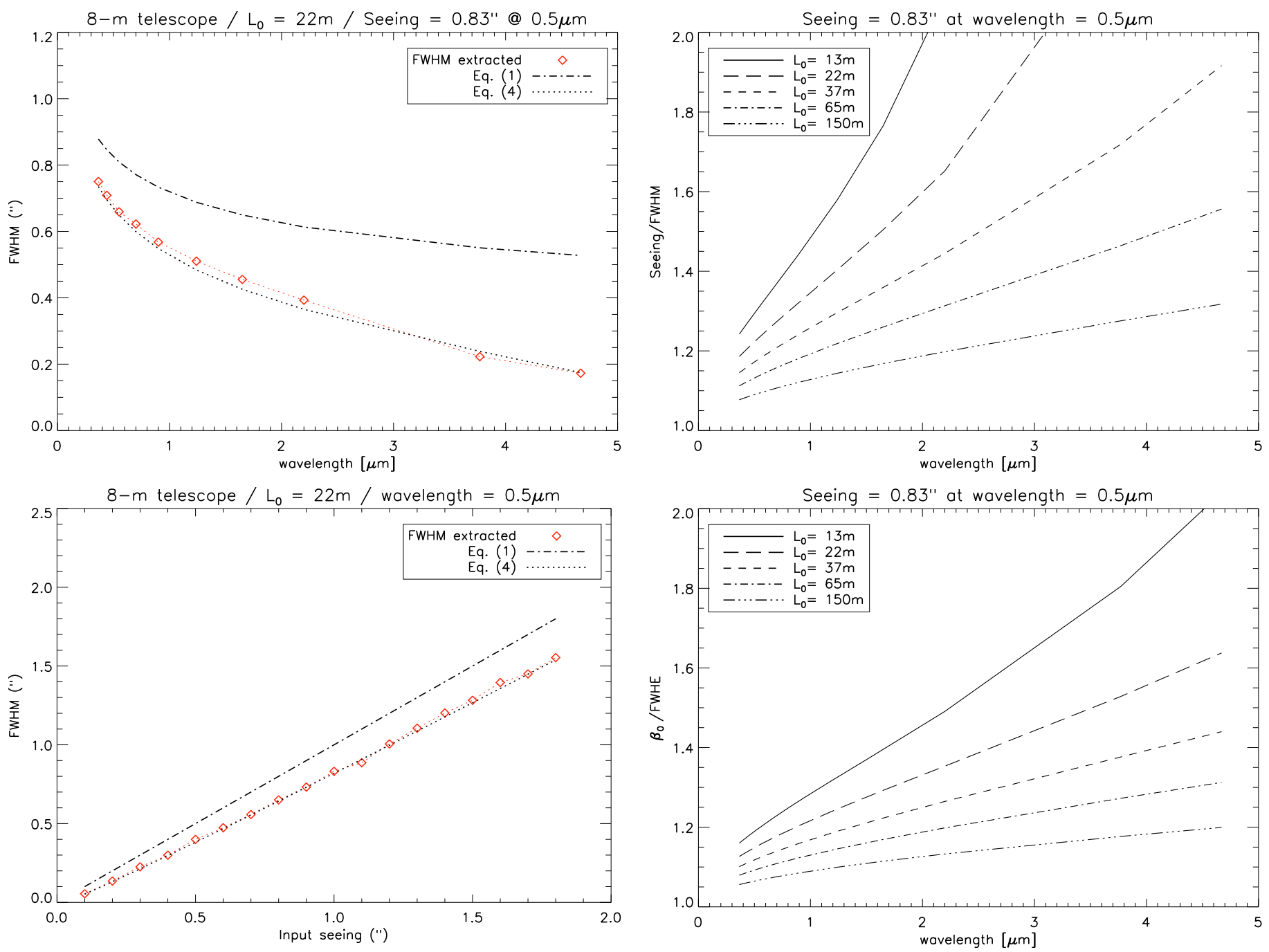

Fig. 3. Dependence of $\theta_{a}$ on wavelength (top, $\left.\varepsilon_{0}=0.83^{\prime \prime}\right)$ and seeing (bottom, $\lambda=0.5 \mu \mathrm{m}$ ). Other parameters: $L_{0}=22 \mathrm{~m}, D=8 \mathrm{~m}$.

finite outer scale. As expected, the validity of Eq. (4) is confirmed, except for the small $D$ where our treatment of diffraction is too crude. All these cases correspond to $L_{0} / r_{0}>80$ where the effect of the finite $L_{0}$ is still mild.

\subsection{Wavelength and seeing dependence}

For the second series of simulations, we considered an 8-m telescope, a fixed outer scale $L_{0}=22 \mathrm{~m}$, and $0.83^{\prime \prime}$ seeing at $0.5 \mu \mathrm{m}$, while the imaging wavelength was allowed to vary from the $U$ band to the $M$-band (from 0.365 to $4.67 \mu \mathrm{m}$ ). The results are presented in the top panel of Fig. 3. We note the stronger dependence of $\theta_{\mathrm{a}}$ on wavelength, compared to the Kolmogorov case. The third series of simulation considered the same $L_{0}$ and $D$, as the seeing conditions were allowed to vary (Fig. 3, bottom). The agreement with Eq. (4) is demonstrated for both wavelength $\left(L_{0} / r_{0}>10\right)$ and seeing dependence $\left(L_{0} / r_{0}>20\right)$.

\subsection{Discussion}

The previous subsections have provided general results about the atmospheric FWHM in the presence of a finite outer scale. To relate these results to true situations, we discuss the particular case

Fig. 4. Top: ratio of seeing $\varepsilon_{0}$ to $F W H M\left(\varepsilon_{\mathrm{vK}}\right)$ as a function of the wavelength for several $L_{0}$ cases. Bottom: similarly, ratio of $\beta_{0}$ to FWHE $\left(\beta_{\mathrm{vK}}\right)$. For both plots, seeing is $0.83^{\prime \prime}$ at $0.5 \mu \mathrm{m}$.

of the 8-m Very Large Telescope at Paranal (Chile) assuming standard seeing conditions $\left(0.83^{\prime \prime}\right.$ at $\left.0.5 \mu \mathrm{m}\right)$, and median outer scale value $\left(L_{0}=22 \mathrm{~m}\right.$, i.e. $\left.L_{0} / r_{0}=180\right)$. Results shown in Fig. 3 indicate that the $F W H M$ of the von Kármán PSF is lower by $19 \%$ than for standard theory $\left(\varepsilon_{0}\right)$ in the visible. This difference is even more dramatic in the near-IR, where the FWHM $\left(\varepsilon_{\mathrm{vK}}\right)$ is lower by $29.7 \%$ ( $H$-band) and $36.3 \%$ ( $K$-band).

In the same way, Fig. 4 (top) quantifies the ratio of $\varepsilon_{0}$ to $\varepsilon_{\mathrm{VK}}$ (i.e., Eq. (1) to (4)) but for several $L_{0}$ values ranging from the Paranal median value (including the 1- $\sigma$ outer scale values, 13 and $37 \mathrm{~m}$ ) to $150 \mathrm{~m}$. The difference with the standard Kolmogorov seeing formula is substantial and can exceed a factor of two in the IR. Likewise, Fig. 4 (bottom) compares the ratio of $\beta_{0}$ to $\beta_{\mathrm{vK}}$.

\section{Resolution under partial compensation}

In analogy with the finite outer scale impact, we discuss here the consequences of a reduction in the low-frequency content of the phase perturbation spectrum generated by AO partial correction or tip-tilt compensation. 
The purpose of adaptive optics (AO) systems is to compensate for the effect of atmospheric wavefront distortions and to reach a diffraction-limited resolution. To do this, the actuator spacing $d$ (or an equivalent measure of $\mathrm{AO}$ compensation order) must be on the order of $2 r_{0}$ or smaller (Roddier 1998). However, AO systems that do not fulfill this condition may still improve the resolution. A good example is the use of the low-order AO system PUEO for observations at visible wavelengths (Rigaut et al. 1998). A resolution gain of up to a factor of two has been reported. To our knowledge, the reduction of the atmospheric PSF under partial compensation has not been explored in a systematic way.

Residual wavefronts after AO compensation contain highfrequency ripple, whereas the perturbations at spatial frequencies lower than $f_{c}=1 /(2 d)$ are corrected. This can be modeled by performing high-pass filtering of the atmospheric PSD. The form of this filter varies, depending on the AO system. The calculations here only illustrate the principle and should be adapted to each AO system if an exact result is sought. We model the AO compensation with a multiplicative factor $F$

$F(x)=x /(1+x), \quad x=\left(|\boldsymbol{f}| / f_{c}\right)^{m}$

where $m=6$. The PSD (Eq. (2)) is multiplied by $F$, the SF is calculated and used to compute the residual PSF in the same way as for the vK spectrum. The SF saturates at $r \gg d$, reaching the value $2 \sigma_{\phi}^{2}=0.62\left(d / r_{0}\right)^{5 / 3}$. The shape of the SF and the saturation value depend on the filter $F(x)$. We experimented with several filters and chose Eq. (7) with $m=6$ because it matches approximately the known formula $\sigma_{\phi}^{2}=0.35\left(d / r_{0}\right)^{5 / 3}$ of Roddier (1998). Comparing this to the saturation level of the vK SF, given by $0.17\left(L_{0} / r_{0}\right)^{5 / 3}$, we find that the effective outer scale of the residual wavefront is $\sim 2 d$.

Once the SF saturates at $2 \sigma_{\phi}^{2}$, the atmospheric OTF reaches a constant level $T_{\min }=\exp \left(-\sigma_{\phi}^{2}\right)$. We can represent such an OTF as a sum of the constant term and a decreasing part. This corresponds to the sum of a diffraction-limited PSF scaled by $S=\exp \left(-\sigma_{\phi}^{2}\right)$ and a wide residual halo. The shape of the halo can therefore be calculated by replacing the atmospheric OTF with $\left(T_{a}-T_{\min }\right) /\left(1-T_{\min }\right)$ out to the distance where the minimum $T_{\min }$ is reached, and setting it to zero for higher frequencies. We note that we renormalize the halo OTF to one at the coordinate origin.

The $F W H M \theta_{\mathrm{AO}}$ and the diameter of a circle containing half the energy $(F W H E)$ were computed for the halo of partially compensated PSFs. We compare these parameters to the noncompensated (Kolmogorov) PSFs in Fig. 5. Even when the actuator spacing $d$ is much larger than $r_{0}$ and the AO system does not perform well in the classical sense $(S \approx 0)$, the gains in $F W H M$ and $F W H E$ are already substantial. Maximum resolution gain $\varepsilon_{0} / \theta_{\mathrm{AO}}$ is reached at $d / r_{0} \sim 4$, when the coherent PSF core is still very weak. As the compensation order increases further, an increasing fraction of energy goes into the core, and the PSF halo becomes weaker and wider. At small $d$ and high $S$, the halo becomes even wider than the uncompensated atmospheric PSF, being produced by residual phase errors on spatial scales smaller than $r_{0}$.

Tip-tilt correction is a particular case of low-order AO compensation. It is well known that maximum resolution gain is achieved at $D / r_{0} \sim 3.6$ (Fried 1966). The gain studied here does not depend on the telescope diameter $D$, but rather on the dimensionless parameter $d / r_{0}$, in full analogy with the effect of the outer scale.

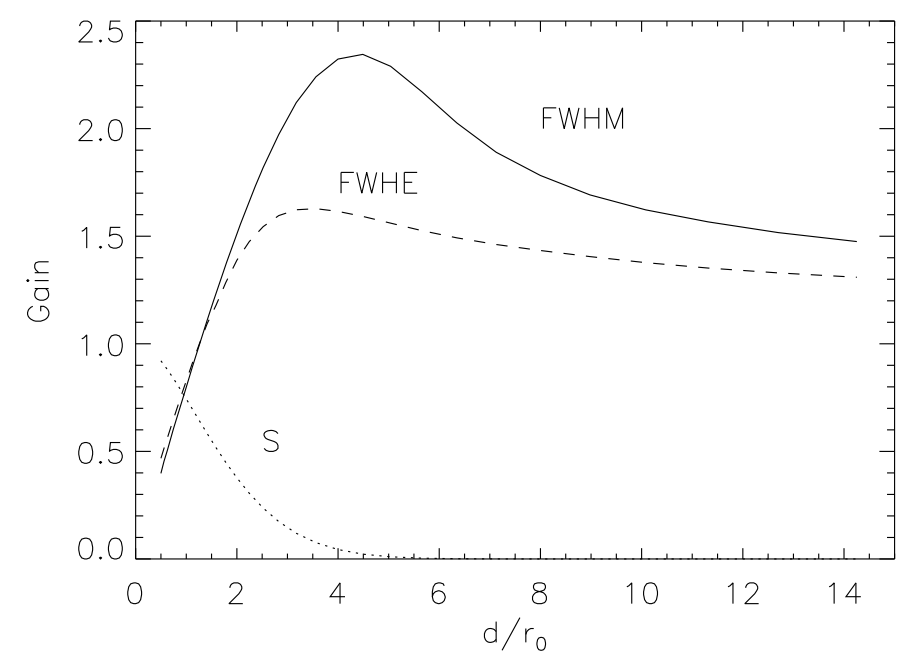

Fig. 5. Gain in the FWHM (full line) and FWHE (dotted line) diameter of the PSF halo (compared to the Kolmogorov PSF) resulting from partial AO compensation (outer scale not included). The dotted line shows the coherent energy $S$.

All three effects - outer scale, partial AO correction and tip-tilt compensation - reduce the low-frequency content of the phase perturbation spectrum. When they act together, the gain in resolution over Kolmogorov turbulence is not cumulative. For example, at finite outer scale the tip-tilt fluctuations become smaller and their correction achieves a smaller resolution gain. The resolution gain from partial AO correction (Fig. 5) is also smaller because of the finite $L_{0}$.

\section{Conclusions and discussion}

This study has been largely motivated by the confusion between seeing and the FWHM of long-exposure images in large telescopes, also often called delivered image quality (DIQ). In an ideal large telescope (no aberrations, internal turbulence, and wind shake), the DIQ is always lower than predicted by the standard theory, owing to the finite turbulence outer scale.

The seeing is usually measured by the Differential Image Motion Monitors (Martin 1987; Sarazin \& Roddier 1990, DIMMs). This method is sensitive to small-scale wavefront distortions and provides estimates of $r_{0}$ that are almost independent of $L_{0}$ (Ziad et al. 1994) ${ }^{1}$. Using the standard theory, we overestimate the $F W H M$ expected for a large telescope. As the PSF is broadened by non-atmospheric factors, this mismatch can hide telescope defects. This is particularly a problem in the IR, where the difference from the standard theory is large. Therefore, before achieving a truly seeing-limited telescope performance in the IR, we must take account of the finite $L_{0}$. Stated in other words, our telescopes could perform better than we predict them to based on the standard theory and DIMM measurements.

On the other hand, If we wish to deduce atmospheric seeing from the width of the long-exposure PSF, the situation is reversed. The true seeing is poorer than we predict. The effect of finite $L_{0}$ is apparent for all telescope diameters. A simultaneous measurement of $L_{0}$ is thus required to be accurate. Estimating seeing from the width of the spots in active-optics

\footnotetext{
1 Seeing monitors based on the absolute image motion are affected by finite $L_{0}$, giving biased, larger $r_{0}$ values.
} 
Shack-Hartmann sensor (long exposures) should be performed with these considerations in mind.

Since internal telescope defects and outer scale act in opposite directions, they can partially compensate for each other. An agreement between DIMM measurements and DIQ can thus be found where it should not be (Sarazin \& Roddier 1990). By comparing simultaneous PSFs at visible and mid-IR wavelengths, it is possible to extract two parameters, $\varepsilon_{0}$ and $L_{0}$, assuming that the telescope's contribution to the image degradation can be neglected (Tokovinin et al. 2007).

\section{References}

Conan, R. 2000, Ph.D. Thesis, Univ. Nice

Consortini, A., Fidanzati, G., Mariani, A., \& Ronchi, L. 1972, Appl. Opt., 11, 1229
Fried, D. L. 1966, J. Opt. Soc. Am. (1917-1983), 56, 1372 Goodman, J. W. 1985, Statistical Optics, ed. J. W. Goodman Kolb, J. 2005, Ph.D. Thesis, Univ. Paris VII

Martin, H. M. 1987, PASP, 99, 1360

McGlamery, B. L. 1976, Computer simulation of atmospheric turbulence and compensated imaging systems

Rigaut, F., Salmon, D., Arsenault, R., et al. 1998, PASP, 110, 152

Roddier, F. 1981, in Progress in optics (Amsterdam, North-Holland: Publishing Co.), ed. E. Wolf, 19, 281

Roddier, F. 1998, Adaptive Optics (Cambridge Univ. Press)

Sarazin, M., \& Roddier, F. 1990, A\&A, 227, 294

Tatarskii. 1961, Dover Publ., Inc., New York

Tokovinin, A. 2002, PASP, 114, 1156

Tokovinin, A., Sarazin, M., \& Smette, A. 2007, MNRAS, 378, 701

Young, A. T. 1974, ApJ, 189, 587

Ziad, A., Bornino, J., Martin, F., \& Agabi, A. 1994, A\&A, 282, 1021

Ziad, A., Conan, R., Tokovinin, A., Martin, F., \& Borgnino, J. 2000, Appl. Opt., 39,5415 